\title{
The negative and detrimental effects of high fructose on the liver, with special reference to metabolic disorders
}

This article was published in the following Dove Press journal: Diabetes, Metabolic Syndrome and Obesity: Targets and Therapy

\section{Brandon H Mai Liang-Jun Yan}

Department of Pharmaceutical Sciences, UNT System College of Pharmacy, University of North Texas Health Science Center, Fort Worth, TX 76107, USA

Correspondence: Liang-Jun Yan Department of Pharmaceutical Sciences, UNT System College of Pharmacy, University of North Texas Health Science Center, 3500 Camp Bowie Blvd, Fort Worth, TX 76107, USA

Tel + I 8177352386

Fax +I 8177352603

Email liang-jun.yan@unthsc.edu

\begin{abstract}
The increased consumption of fructose in the average diet through sweeteners such as high-fructose corn syrup (HFCS) and sucrose has resulted in negative outcomes in society through producing a considerable economic and medical burden on our healthcare system. Ingestion of fructose chronically has contributed to multiple health consequences, such as insulin resistance, obesity, liver disorders, and diabetes. Fructose metabolism starts with fructose phosphorylation by fructose kinase in the liver, and this process is not feedback regulated. Therefore, ingestion of high fructose can deplete ATP, increase uric acid production, and increase nucleotide turnover. This review focuses the discussion on the hepatic manifestations of high fructose-implicated liver metabolic disorders such as insulin resistance, obesity due to enhanced lipogenesis, non-alcoholic fatty liver disease (NAFLD), nonalcoholic steatohepatitis (NASH), and type 2 diabetes. The detrimental effects of high fructose on the liver, contributed potentially by microbiome and leptin, were also discussed. The authors believe that, together with diet management, further studies focusing on disrupting or blocking fructose metabolism in the liver may help with designing novel strategies for prevention and treatment of fructose-induced chronic liver metabolic diseases.
\end{abstract}

Keywords: fructose, liver, metabolic syndrome, non-alcoholic fatty liver disease, nonalcoholic steatohepatitis

\section{Introduction}

Morbidity and mortality associated with chronic metabolic syndrome have been on the rise in the US for the last 30 years. ${ }^{1}$ It has been estimated that nearly $60 \%$ of the adult population in the US is overweight, with prevalence of insulin-dependent type 2 diabetes standing currently at approximately $9.3 \% .{ }^{1}$ Moreover, the past 10 years has witnessed increased death from liver dysfunction, cardiovascular disease, and certain forms of cancer that are associated with obesity and type 2 diabetes. ${ }^{2}$ Therefore, the economic, psychological, and social burdens have been of major concerns nowadays; and there has been increasing interest in studying the pathological mechanisms of chronic metabolic diseases, in the hopes to find effective preventive and treatment strategies.

It is now generally thought that increased consumption of added sugar, in particular fructose sweeteners, ${ }^{3-6}$ is one of the major underlying causes of chronic metabolic diseases, including obesity, insulin resistance, non-alcoholic fatty liver disease (NAFLD), diabetes, and cardiovascular diseases. ${ }^{7-12}$ In this review, we will focus on the effects of high fructose on the liver, with special reference to metabolic disorders. 


\section{Fructose in diet and healthcare burden}

Fructose is classified as a simple sugar and is found naturally in the diet in the form of fruits or honey. However, its consumption has increased drastically over the last few centuries due to the average diet's increased intake of sweeteners that are highly made up of fructose, including high-fructose corn syrup (HFCS) and sucrose. ${ }^{5,7}$ These sweeteners are especially prevalent in many sugary beverages such as soft drinks, as well as candies. Fructose is dangerous because its sweetening effect is 2-times higher than that of glucose, which can cause people to crave more fructose-containing soft drinks. Chronic fructose ingestion, most notably in industrialized countries, has contributed to increasing prevalence of obesity and a myriad of chronic health conditions, including those involved with metabolic syndrome, ${ }^{7,13,14}$ that have produced a substantial economic and medical burden on our healthcare system. ${ }^{13,15}$ In particular, the hepatic consequences of metabolic syndrome have led to negative outcomes and continue to be a subject matter of interest. ${ }^{6,16,17}$

\section{Fructose consumption and chronic metabolic liver disorders}

It has been found that consumption of high fructose, along with other sugars such as sucrose, increases the risk of both NAFLD and non-alcoholic steatohepatitis (NASH). ${ }^{18,19}$ In fact, a study that involved patients being administered sugary beverages containing high fructose for a duration of 6 months found that the patients had shown increases of fat in the liver by the end of the study. ${ }^{5}$ Some studies' findings also suggested that consumption of less sugary beverages and overall fructose intake may allow for less accumulation of fat in the liver. A clear correlation between fructose intake and the prevalence of obesity and NAFLD has been found based on epidemiological data. ${ }^{19}$ The complications that can arise from NAFLD include chronic liver disease and cirrhosis, with the severity of liver fibrosis being manifested as a dose-dependent manner of fructose intake. ${ }^{19}$

\section{Fructose consumption, lipogenesis, uric acid production, and insulin resistance}

Although it has been known that fructose can be converted to both plasma and liver triglyceride (TG), this has been found to not be the major factor contributing to fatty liver. Instead, fat accumulation primarily occurs in the liver as a result of fructose metabolism in the liver causing impaired beta oxidation of fatty acids in the liver and enhanced de novo lipogenesis. ${ }^{5}$ Recent studies have found that increased expression of fructokinase $\mathrm{C}$ (also referred to as ketohexokinase) plays a major role in this fat accumulation via a decrease of ATP, uric acid generation, and nucleotide turnover. ${ }^{5}$ The metabolism of fructose is characterized by use of ATP by fructokinase C to convert fructose to fructose-1-phosphate, which is then metabolized to dihydroxyacetone phosphate and D-glyceraldehyde by aldolase $\mathrm{B}$ (Figure 1). ${ }^{5}$ As the metabolism progresses through various other steps, the end result of this pathway is the production of glycogen, glucose, and TG. However, the decrease in ATP that occurs as fructokinase $\mathrm{C}$ phosphorylates fructose results in activation of adenosine monophosphate (AMP) deaminase, which results in transformation of AMP to inosine monophosphate (IMP). This results in nucleotide turnover and subsequent uric acid generation. ${ }^{5}$ The consequences of this fall in ATP and elevation in uric acid include oxidative stress, inhibition of protein synthesis, and mitochondrial dysfunction, which can further lead to obesity, insulin resistance, and fatty liver, eventually culminating in metabolic syndrome. Furthermore, fructokinase may also cause hepatic insulin resistance, which can contribute to metabolic syndrome and further progression of NAFLD. ${ }^{15,20}$ It should be highlighted that insulin resistance caused by high fructose ingestion is likely due to the accumulation of serum uric acid, which can increase oxidative stress and blunt insulin sensitivity. ${ }^{21}$ Moreover, induction of insulin resistance and diabetes may also be caused via hepatic/pancreatic endoplasmic reticulum stress. ${ }^{22}$

Uric acid generation, in particular, has been found to lead to hepatic lipogenesis via several potential pathways. Its activation of NADPH oxidase and oxidative stress was shown to decrease the activity of the mitochondrial enzyme known as aconitase-2, which results in citrate accumulation and activation of ATP citrate lyase that leads to lipogenesis. ${ }^{5}$ Another possible mechanism is via uric acid's impairment of fatty acid oxidation through the inhibition of AMP-activated protein kinase (AMPK), which leads to enoyl CoA hydratase inhibition and lipid build-up. ${ }^{5}$ Depletion of ATP stores in the liver is also a consequence of this process. As a result, studies have shown that hyperuricemia is associated with both NAFLD and NASH. Uric acid may 


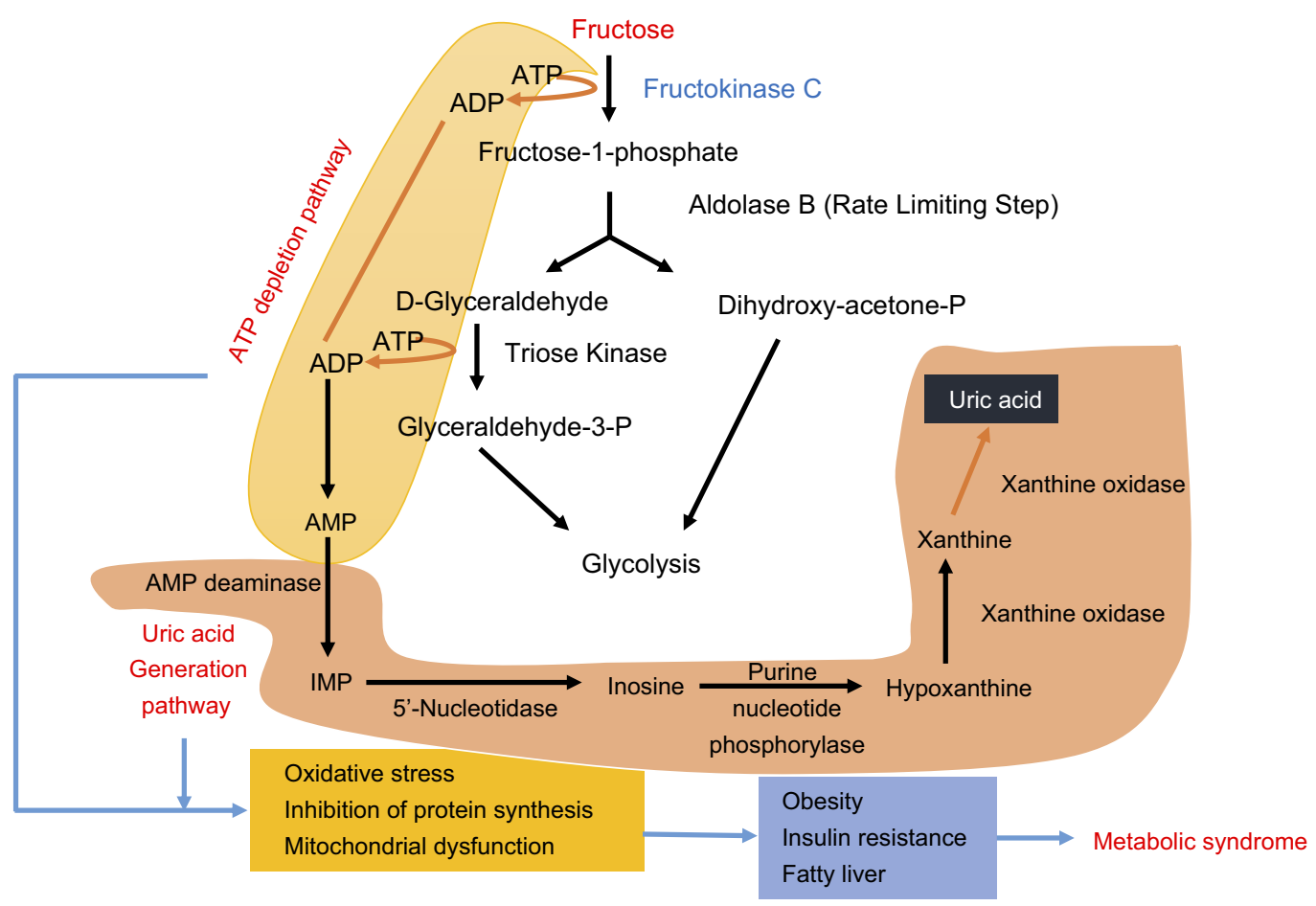

Figure I Fructose metabolism via fructokinase. This pathway is uncontrolled and can deplete ATP and is involved in uric acid production (as highlighted in the figure). Consequently, the fall in ATP and increase in uric acid can cause oxidative stress, inhibition of protein synthesis, and mitochondrial dysfunction, which can further induce obesity, insulin resistance, and fatty liver that eventually manifest in global metabolic syndrome.

also enhance the effects of fructose via a positive feedback system which leads to stimulation of aldose reductase and subsequent fructose generation, as well as increasing activity of fructokinase which leads to further fructose metabolism. ${ }^{5}$

\section{Controversies regarding fructose consumption and serum uric acid levels}

It should be pointed out that there are some controversial reports in the literature regarding the link between high fructose intake and increased fasting serum uric acid. For example, under isocaloric or energy-matched conditions, there was no effect of high fructose on levels of fasting serum uric acid. ${ }^{23}$ This observation, however, has been thought to be due to the design of the experiment, whereby the initial increase in uric acid content is transient and lasts only for a short duration. ${ }^{23}$ Indeed, continued ingestion of high fructose over the long-term would induce a chronic increase in fasting serum uric acid. ${ }^{23}$ It should be born in mind that the most important factor involved in metabolic syndrome is the total amount of calories ingested every day rather than the composition of the diet.

\section{Effects of fructokinase C, microbiome, and leptin on fructose metabolism}

Actions of fructokinase $\mathrm{C}$ in the small intestine are also thought to induce the formation of fatty liver. Studies have found that fructokinase $\mathrm{C}$ phosphorylation of fructose in the gut interferes with the tight junctions and, thus, increases permeability in the gut, which subsequently causes the introduction of endotoxin into the portal vein that facilitates production of fatty liver. ${ }^{5}$ This endotoxemia may also result in inflammation, which also plays a role in NAFLD development. Moreover, alteration of the normal gut microbiome which is induced by fructose further contributes to NAFLD progression. ${ }^{15,24}$ This is probably due to dysregulation in the conversion of primary bile acids to secondary bile acids in the small intestine by fructose-altered microbiome, leading to decreased activation of several nuclear receptors that are involved in energy regulation. ${ }^{25}$ Dysregulation of these nuclear receptors, therefore, could cause NAFLD. ${ }^{25}$ It should be noted that fructose can also be endogenously produced in diabetic individuals via the polyol pathway, ${ }^{26-29}$ and both exogenous and endogenous fructose can coat proteins via post-translational modification mechanisms ${ }^{30,31}$ that further accentuate metabolic stress in 
the liver. ${ }^{29,32,33}$ Additionally, in terms of an association with metabolic stress, fructose and leptin are closely related. ${ }^{34}$ For example, long-term fructose ingestion evokes leptin resistance and stimulates leptin production by gastric mucosa. ${ }^{35}$ Furthermore, leptin increases the activity of the fructose transport GLUT-5, thereby increasing fructose uptake. $^{36}$

\section{Controversies regarding the relationship between high fructose and development of NAFLD}

With respect to the relationship between high fructose and the development of NAFLD, there has been a report of large human cohort studies where consumption of high fructose is inversely associated with NAFLD. ${ }^{37}$ This observation is likely due to the fact that the source of fructose, be it from fruits or from soft drinks, was not separated in this study. ${ }^{37}$ This could also be true in other human trials whereby high fructose consumption failed to cause obesity or metabolic syndrome. ${ }^{38-41}$ These studies indicate that there is a big difference between fructose from natural fruits and fructose from soft drinks, as the former contains a variety of chemical compounds including minerals, vitamins, antioxidants, and polyphenols that could block high fructose effects. ${ }^{21}$

\section{Summary}

In summary, the actions and possible detrimental effects of high fructose on the liver are concerning, especially with the rise in consumption of sweeteners such as HFCS and sucrose. Its contributions to NAFLD and NASH have been supported by numerous studies, and can be explained by multiple mechanisms. Among the potential causes found, impaired beta oxidation of fatty acids in the liver and enhanced de novo lipogenesis are thought to contribute most towards this outcome. Fructokinase $\mathrm{C}$ has been especially noted to facilitate this fat accumulation in the liver via uric acid generation, a decrease of ATP, and increased nucleotide turnover. Still, there are contradictory findings in some studies that suggest fructose consumed over shorter durations or in lower doses may not express these relationships. Additionally, it should be noted that there are likely many other factors that contribute towards the magnitude of the effects of fructose which should be taken into consideration, such as physical inactivity and other dietary components, as well as gestation and lactation periods which lead to deep metabolic changes in mothers and in their progeny via metabolic early programming. For instance, it has been reported that severe hepatic damage occurred in dams and in their offspring after a high fructose diet. $^{42,43}$ Further research with larger clinical trials that examine the effects of lowering HCFS and sugar consumption as well as uric acid levels is warranted to confirm the impact of fructose on health. Moreover, further investigation to gain a better understanding of inhibiting or disrupting fructose metabolism in the liver and its effects on specific metabolic regulators may potentially lead to innovative solutions towards chronic liver disorder prevention and treatment that can change the landscape of healthcare.

\section{Acknowledgments}

The writing of this article was supported in part by University of North Texas Health Science Center intramural grants RI 10015 (LJY) and RI10039 (LJY) and by the National Institutes of Health grant R01NS079792 (LJY). This paper is the outcome of a special topics in pharmacy research course (PHAR 7375) offered by the corresponding author in the College of Pharmacy at University of North Texas Health Science Center.

\section{Disclosure}

The authors report no conflicts of interest in this work.

\section{References}

1. Vreman RA, Goodell AJ, Rodriguez LA, Porco TC, Lustig RH, Kahn JG. Health and economic benefits of reducing sugar intake in the USA, including effects via non-alcoholic fatty liver disease: a microsimulation model. BMJ Open. 2017;7(8):e013543. doi:10.1136/bmjopen-2016-013543

2. Ter Horst KW, Serlie MJ. Fructose consumption, lipogenesis, and non-alcoholic fatty liver disease. Nutrients. 2017;9(9):E981. doi: 10.3390/nu9090981

3. Delbridge LM, Benson VL, Ritchie RH, Mellor KM. Diabetic cardiomyopathy: the case for a role of fructose in disease etiology. Diabetes. 2016;65(12):3521-3528. doi:10.2337/db16-0682

4. Softic S, Cohen DE, Kahn CR. Role of dietary fructose and hepatic de novo lipogenesis in fatty liver disease. Dig Dis Sci. 2016;61 (5):1282-1293. doi:10.1007/s10620-016-4054-0

5. Jensen T, Abdelmalek MF, Sullivan S, et al. Fructose and sugar: A major mediator of non-alcoholic fatty liver disease. $J$ Hepatol. 2018;68(5):1063-1075. doi:10.1016/j.jhep.2018.01.019

6. DiNicolantonio JJ, Subramonian AM, O'Keefe JH. Added fructose as a principal driver of non-alcoholic fatty liver disease: a public health crisis. Open Heart. 2017;4(2):e000631. doi:10.1136/openhrt-2017-000631

7. Jegatheesan P, De Bandt JP. Fructose and NAFLD: the multifaceted aspects of fructose metabolism. Nutrients. 2017;9:3.

8. Tran LT, Yuen VG, McNeill JH. The fructose-fed rat: a review on the mechanisms of fructose-induced insulin resistance and hypertension. Mol Cell Biochem. 2009;332(1-2):145-159. doi:10.1007/s11010-0090184-4 
9. Rippe JM, Angelopoulos TJ. Fructose-containing sugars and cardiovascular disease. Adv Nutr. 2015;6(4):430-439. doi:10.3945/ an. 114.008177

10. Tappy L. Fructose-containing caloric sweeteners as a cause of obesity and metabolic disorders. J Exp Biol. 2018;221(PtSuppl 1). doi:10.1242/ jeb. 164202

11. Tappy L. Fructose metabolism and noncommunicable diseases: recent findings and new research perspectives. Curr Opin Clin Nutr Metab Care. 2018;21(3):214-222. doi:10.1097/MCO.0000000000000460

12. Macdonald IA. A review of recent evidence relating to sugars, insulin resistance and diabetes. Eur $J$ Nutr. 2016;55(Suppl 2):17-23. doi:10.1007/s00394-016-1340-8

13. Lirio LM, Forechi L, Zanardo TC, et al. Chronic fructose intake accelerates non-alcoholic fatty liver disease in the presence of essential hypertension. $J$ Diabetes Complications. 2016;30(1):85-92. doi:10.1016/j.jdiacomp.2015.10.008

14. Alwahsh SM, Gebhardt R. Dietary fructose as a risk factor for non-alcoholic fatty liver disease (NAFLD). Arch Toxicol. 2017;91 (4):1545-1563. doi:10.1007/s00204-016-1892-7

15. Bantle JP. Dietary fructose and metabolic syndrome and diabetes. J Nutr. 2009;139(6):1263S-1268S. doi:10.3945/jn.108.098020

16. Chiu S, Mulligan K, Schwarz JM. Dietary carbohydrates and fatty liver disease: de novo lipogenesis. Curr Opin Clin Nutr Metab Care. 2018;21(4):277-282. doi:10.1097/MCO.0000000000000469

17. Nobili V, Mosca A, De Vito R, Raponi M, Scorletti E, Byrne CD. Liver zonation in children with non-alcoholic fatty liver disease: associations with dietary fructose and uric acid concentrations. Liver Int. 2018;38(6):1102-1109. doi:10.1111/liv.13661

18. Choi Y, Abdelmegeed MA, Song BJ. Diet high in fructose promotes liver steatosis and hepatocyte apoptosis in C57BL/6J female mice: role of disturbed lipid homeostasis and increased oxidative stress. Food Chem Toxicol. 2017;103:111-121. doi:10.1016/j.fct.2017.02.039

19. Ouyang X, Cirillo P, Sautin Y, et al. Fructose consumption as a risk factor for non-alcoholic fatty liver disease. J Hepatol. 2008;48 (6):993-999. doi:10.1016/j.jhep.2008.02.011

20. Warren BE, Lou PH, Lucchinetti E, et al. Early mitochondrial dysfunction in glycolytic muscle, but not oxidative muscle, of the fructose-fed insulin-resistant rat. Am J Physiol Endocrinol Metab. 2014;306(6):E658-E667. doi:10.1152/ajpendo.00511.2013

21. Johnson RJ, Nakagawa T, Sanchez-Lozada LG, et al. Sugar, uric acid, and the etiology of diabetes and obesity. Diabetes. 2013;62 (10):3307-3315. doi:10.2337/db12-1814

22. Balakumar M, Raji L, Prabhu D, et al. High-fructose diet is as detrimental as high-fat diet in the induction of insulin resistance and diabetes mediated by hepatic/pancreatic endoplasmic reticulum (ER) stress. Mol Cell Biochem. 2016;423(1-2):93-104. doi:10.1007/s11010-016-2828-5

23. Wang DD, Sievenpiper JL, de Souza RJ, et al. The effects of fructose intake on serum uric acid vary among controlled dietary trials. J Nutr. 2012;142(5):916-923. doi:10.3945/jn.111.151951

24. Lambertz J, Weiskirchen S, Landert S, Weiskirchen R. Fructose: a dietary sugar in crosstalk with microbiota contributing to the development and progression of non-alcoholic liver disease. Front Immunol. 2017;8:1159. doi:10.3389/fimmu.2017.01159

25. Chen J, Thomsen M, Vitetta L. Interaction of gut microbiota with dysregulation of bile acids in the pathogenesis of nonalcoholic fatty liver disease and potential therapeutic implications of probiotics. $J$ Cell Biochem. 2019;120(3):2713-2720. doi:10.1002/jcb.27635

26. Wu J, Jin Z, Zheng H, Yan LJ. Sources and implications of NADH/ $\mathrm{NAD}(+)$ redox imbalance in diabetes and its complications. Diabetes Metab Syndr Obes. 2016;9:145-153. doi:10.2147/DMSO.S106087

27. Luo X, Wu J, Jing S, Yan LJ. Hyperglycemic stress and carbon stress in diabetic glucotoxicity. Aging Dis. 2016;7(1):90-110. doi:10.14336/ AD.2015.0702
28. Yan LJ. Pathogenesis of chronic hyperglycemia: from reductive stress to oxidative stress. J Diabetes Res. 2014;2014:137919. doi:10.1155/ 2014/137919

29. Lanaspa MA, Ishimoto T, Li N, et al. Endogenous fructose production and metabolism in the liver contributes to the development of metabolic syndrome. Nat Commun. 2013;4:2434. doi:10.1038/ ncomms 3434

30. Gugliucci A. Formation of fructose-mediated advanced glycation end products and their roles in metabolic and inflammatory diseases. $A d v$ Nutr. 2017;8(1):54-62. doi:10.3945/an.116.013912

31. Takagi Y, Kashiwagi A, Tanaka Y, Asahina T, Kikkawa R, Shigeta Y. Significance of fructose-induced protein oxidation and formation of advanced glycation end product. J Diabetes Complications. 1995;9 (2):87-91.

32. Leung C, Herath CB, Jia Z, et al. Dietary advanced glycation end-products aggravate non-alcoholic fatty liver disease. World J Gastroenterol. 2016;22(35):8026-8040. doi:10.3748/wjg.v22.i35.8026

33. Aragno M, Mastrocola R. Dietary sugars and endogenous formation of advanced glycation endproducts: emerging mechanisms of disease. Nutrients. 2017;9:4. doi:10.3390/nu9040385

34. Sainz N, Barrenetxe J, Moreno-Aliaga MJ, Martinez JA. Leptin resistance and diet-induced obesity: central and peripheral actions of leptin. Metabolism. 2015;64(1):35-46. doi:10.1016/j.metabol. 2014.10.015

35. Aijala M, Malo E, Ukkola O, et al. Long-term fructose feeding changes the expression of leptin receptors and autophagy genes in the adipose tissue and liver of male rats: a possible link to elevated triglycerides. Genes Nutr. 2013;8(6):623-635. doi:10.1007/s12263013-0357-3

36. Sakar Y, Nazaret C, Letteron P, et al. Positive regulatory control loop between gut leptin and intestinal GLUT2/GLUT5 transporters links to hepatic metabolic functions in rodents. PLoS One. 2009;4(11): e7935. doi:10.1371/journal.pone.0007935

37. Kanerva N, Sandboge S, Kaartinen NE, Mannisto S, Eriksson JG. Higher fructose intake is inversely associated with risk of nonalcoholic fatty liver disease in older finnish adults. Am J Clin Nutr. 2014;100(4):1133-1138. doi:10.3945/ajcn.114.086074

38. Chiavaroli L, Ha V, de Souza RJ, Kendall CW, Sievenpiper JL. Fructose in obesity and cognitive decline: is it the fructose or the excess energy? Nutr J. 2014;13:27. doi:10.1186/1475-2891-13-27

39. Tajima R, Kimura T, Enomoto A, et al. No association between fruits or vegetables and non-alcoholic fatty liver disease in middle-aged men and women. Nutrition. 2019;61:119-124. doi:10.1016/j. nut.2018.10.016

40. Ha V, Sievenpiper JL, de Souza RJ, et al. Effect of fructose on blood pressure: a systematic review and meta-analysis of controlled feeding trials. Hypertension. 2012;59(4):787-795. doi:10.1161/ HYPERTENSIONAHA.111.182311

41. Sievenpiper JL, de Souza RJ, Mirrahimi A, et al. Effect of fructose on body weight in controlled feeding trials: a systematic review and meta-analysis. Ann Intern Med. 2012;156(4):291-304. doi:10.7326/ 0003-4819-156-4-201202210-00007

42. Ojeda ML, Nogales F, Munoz Del Valle P, Diaz-Castro J, Murillo ML, Carreras O. Metabolic syndrome and selenium in fetal programming: gender differences. Food Funct. 2016;7 (7):3031-3038. doi:10.1039/c6fo00595k

43. Nogales F, Ojeda ML, Del Valle PM, Serrano A, Murillo ML, Carreras Sanchez O. Metabolic syndrome and selenium during gestation and lactation. Eur J Nutr. 2017;56(2):819-830. doi:10.1007/ s00394-015-1129-1 


\section{Publish your work in this journal}

Diabetes, Metabolic Syndrome and Obesity: Targets and Therapy is an international, peer-reviewed open-access journal committed to the rapid publication of the latest laboratory and clinical findings in the fields of diabetes, metabolic syndrome and obesity research. Original research, review, case reports, hypothesis formation, expert opinion and commentaries are all considered for publication. The manuscript management system is completely online and includes a very quick and fair peer-review system, which is all easy to use. Visit http://www.dovepress.com/testimonials.php to read real quotes from published authors. 